\title{
A SUB-PPB-LEVEL INTEGRATED ELECTROCHEMICAL HEAVY METAL ION MICROSENSOR
}

\author{
Huawei Jiang ${ }^{1}$, Chen Yang ${ }^{2}$, Ken Yang ${ }^{2}$ and Liang Dong ${ }^{2 *}$ \\ ${ }^{1}$ Iowa State University, Ames, Iowa, USA \\ ${ }^{2}$ Analog Devices Inc., Wilmington, MA, USA
}

\begin{abstract}
This paper reports a miniaturized on-chip electrochemical sensor with integrated microchannel and bismuth (Bi) planner electrode for detecting trace amount of $\mathrm{Pb}^{2+}$ ions by anodic stripping voltammetry (ASV). A low limit of detection (LOD) of $0.3 \mathrm{ppb}$ and is achieved. The result is promising for trace heavy metal ion detections in industrial and residential water quality monitoring and treatment applications.
\end{abstract}

\section{INTRODUCTION}

Toxic heavy metal ions $\left(\mathrm{Pb}^{2+}, \mathrm{Cd}^{2+}, \mathrm{Co}^{2+}, \mathrm{Cr}^{2+}\right.$, etc. $)$ possesses severe threats to public health, due to the increasingly serious pollution worldwide. A rapid and accurate detection technology is thus in great demand. Lab tests use high-end instruments like inductively coupled plasma mass spectrometer and ion chromatography are bulky, expensive, time-consuming and limited usage for in situ environmental screening and monitoring, while less-expensive test papers and ion-selective electrodes suffer from severe ion cross-sensitivity, low sensitivity, or short operation lifetime. Therefore, it is of critical importance to develop a highperformance, cost-effective heavy metal ion detection platform for portable applications. Among the effective methods, electrochemical measurement of anodic stripping voltammetric analysis is recognized as the most sensitive electroanalytical technique [1-5].

Anodic stripping voltammetry (ASV) is considered one of the most sensitive methods for trace-amount determination of many metals and compounds in environmental and biological samples.
Various materials have been used to form working electrodes of ASV sensors. Bismuth (Bi) is advantageous over mercury, Au [6], and carbon [7], due to the near-zero toxicity, wide potential window, and insensitivity to dissolved oxygen. It should be noted that several electrochemical heavy metal ion sensors have been reported using an assembled bulk electrochemical cell setup $[8,9]$. In contrast, onchip microsensors require using integrated circuit (IC) compatible materials and fabrications.

Here, we present a miniaturized ASV heavy metal ion sensor utilizing a Bi based thin-film working electrode (WE) formed via conventional evaporation and photolithography (Fig. 1a). Compared to $\mathrm{Bi}$ electrodes formed by in situ method, in which extra $\mathrm{Bi}^{3+}$ ions are added in the analyte solution and then co-electrodeposited with the target analyte metals to compensate for material consumptions [10] during ASV measurements (Fig. 1b), our ex situ Bi electrode will enable rapid test and mass production of sensors. Particularly, the microsensor consists of a $\mathrm{Bi} \mathrm{WE}$, a silver/silver chloride $(\mathrm{Ag} / \mathrm{AgCl})$ reference electrode $(\mathrm{RE})$, and an $\mathrm{Au}$ counter electrode (CE) (Fig. 1c), embedded inside a microfluidic channel for the ASV detection of heavy metal ions in a miniaturized format (Fig. 1d).

\section{EXPERIMENTAL}

Following materials were used to prepare $0.1 \mathrm{M}$ acetate buffer solution $(\mathrm{pH}=4.6)$ : $0.1 \mathrm{M}$ Acetic acid (Fisher Scientific, Fair Lawn, NJ), 0.1 M sodium acetate (Sigma-Aldrich, St. Louis, MO), $0.1 \mathrm{M}$ $\mathrm{NaCl}$ (Fisher Scientific, Fair Lawn, NJ), and deionized (DI) water. All electrochemical measurements were prepared from $1000 \mathrm{ppm}$ $\mathrm{Pb}^{2+}$ (Sigma-Aldrich, St. Louis, $\mathrm{MO}$ ) and $\mathrm{Cd}^{2+}$ (Fisher Scientific,
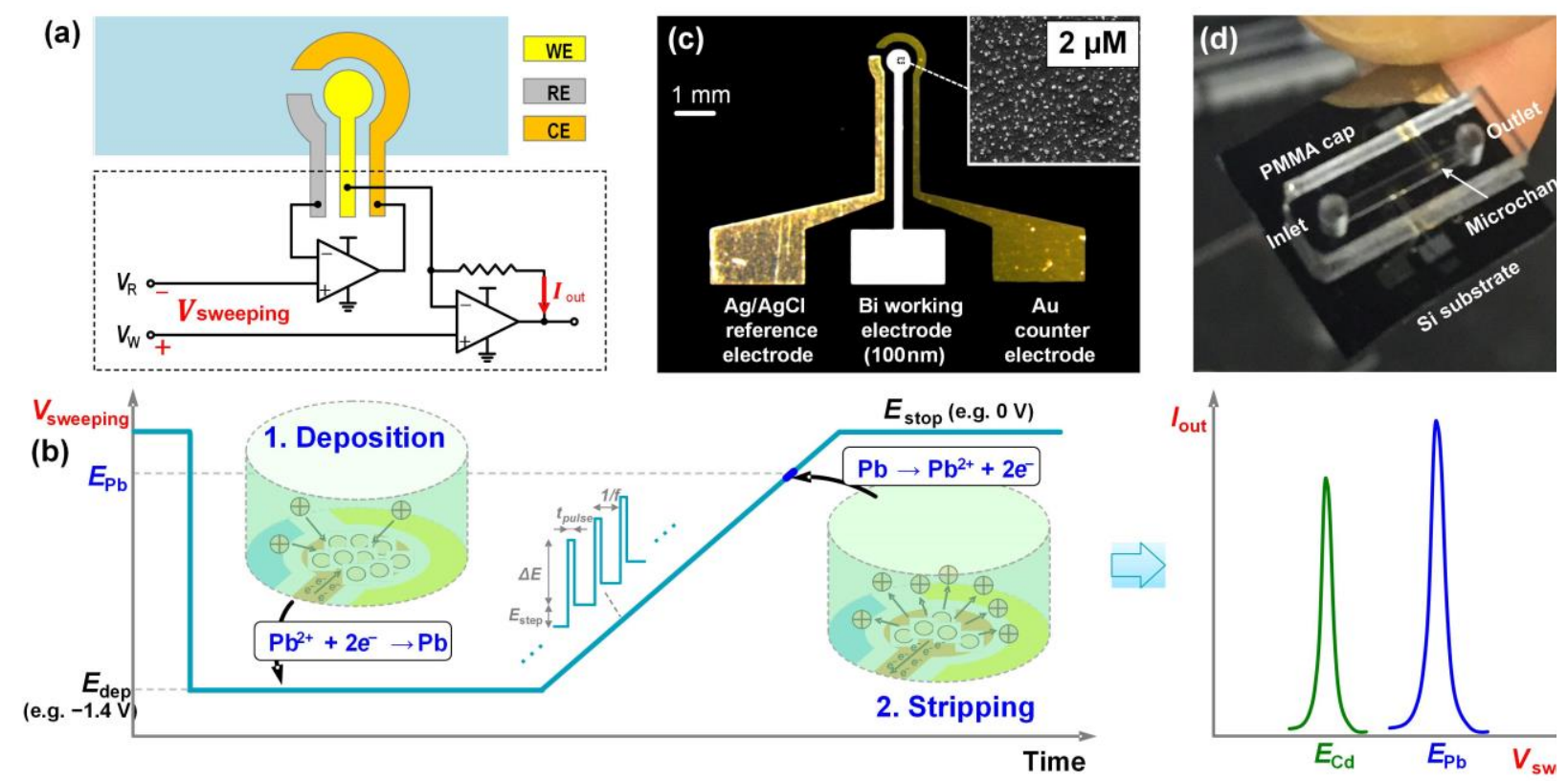

Figure 1: (a) Schematic of the electrochemical sensor and signal reading circuit. (b) Selective anodic stripping voltammetry (ASV) method. (c) Photos of the 3-electrodes prototype. (d) A sensing unit example with two sensors in the microchannel. 
Fair Lawn, NJ) stock standard solution. Necessary dilutions were prepared with acetate buffer solution for various concentration study.

Standard fabrication processes were used to prepare the microsensor. $\mathrm{A} \mathrm{SiO}_{2}$ layer (300 $\mathrm{nm}$ thickness) was first grown on a silicon wafer via thermal oxidation. After a Ti/Au (5/80 nm thickness) layer was deposited on top of $\mathrm{SiO}_{2}$ using e-beam evaporation, three $\mathrm{Ti} / \mathrm{Au}$ electrodes were patterned via photolithography and wet chemical etching. Next, Ti/Bi (5/150 nm thickness) was deposited using ebeam evaporation and then patterned to form a WE on the surface of a Ti/Au electrode using a lift-off process. The same process was employed to form a Ti/Ag (5/500 nm thickness) electrode. Further, the device was immersed in a $0.1 \mathrm{M} \mathrm{FeCl}_{3}$ solution containing 0.1 $\mathrm{M} \mathrm{HCl}$ for $10 \mathrm{~s}$ to form a $\mathrm{Ag} / \mathrm{AgCl} \mathrm{RE}$. Last, a poly(methyl methacrylate) or PMMA microfluidic channel (length: $8 \mathrm{~mm}$; width: $1.5 \mathrm{~mm}$; depth: $0.1 \mathrm{~mm}$ ) was formed using hot embossing and then bonded with the substrate using an ultraviolet adhesive (PermiNex 2010, MicroChem, Westborough, MA). The sensing area of the WE is about $0.2 \mathrm{~mm}^{2}$.

Square wave ASV was used to perform ion measurements. A deposition potential of $-1.4 \mathrm{~V}$ was applied for $30 \mathrm{~s}$, followed by applying a potential scan voltage from -1.4 to $0 \mathrm{~V}$ with an amplitude of $25 \mathrm{mV}$, a pulse width of $50 \mathrm{~ms}$, a potential step of $4 \mathrm{mV}$, and a frequency of $5 \mathrm{~Hz}$.

\section{RESULTS AND DISCUSSION}
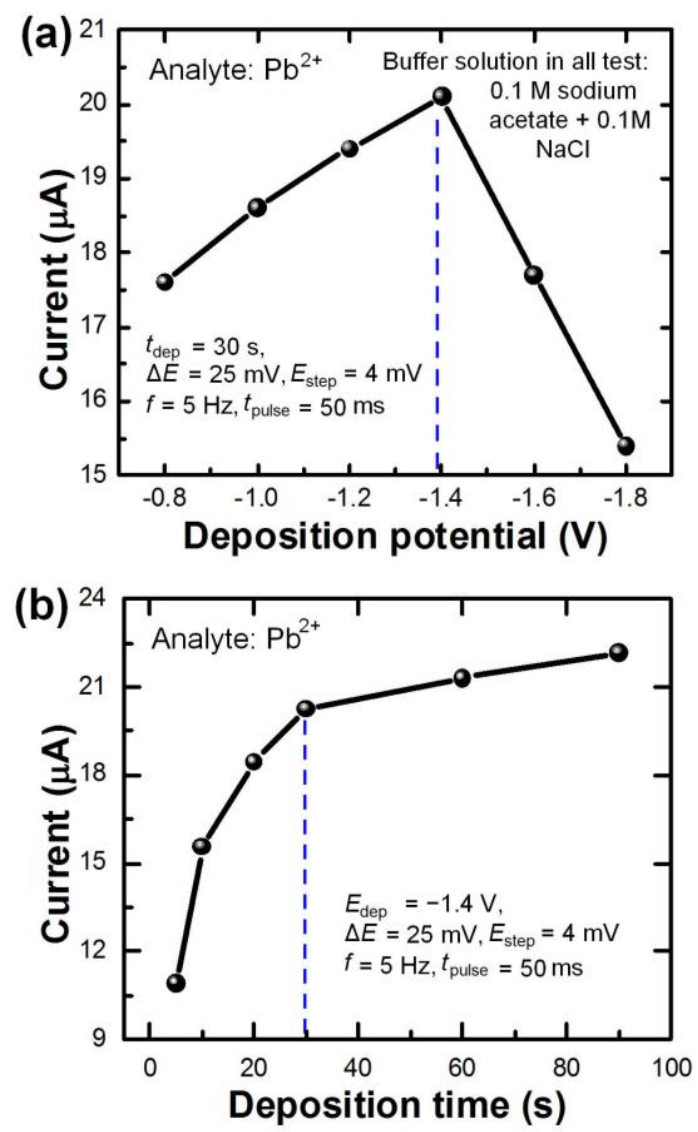

Figure 2: Effect of (a) the deposition potential and (b) deposition time on the stripping peak current of $100 \mathrm{ppb}$ of $\mathrm{Pb}^{2+}$ in acetate buffer ( $\mathrm{pH}$ 4.6).
Fig. 2a shows the effect of deposition potential in a range from

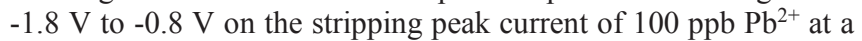
deposition time of $30 \mathrm{~s}$. As the deposition potential positively increased to $-1.4 \mathrm{~V}$, the highest peak current was observed. After that, the peak current declined significantly due to the enhancement of electrolytic hydrogen evolution. Thus, $-1.4 \mathrm{~V}$ was used as the deposition potential in the following electrochemical measurement.

Fig. $2 \mathrm{~b}$ demonstrates the influence of deposition time in a range from $5 \mathrm{~s}$ to $90 \mathrm{~s}$ on the stripping peak current at the $-1.4 \mathrm{~V}$ deposition potential. The peak current increased sharply with increasing deposition time, because of the increased amount of $\mathrm{Pb}^{2+}$. However, the stripping peak current started to increase slowly after the deposition time of $30 \mathrm{~s}$, possibly due to the surface saturation of the WE. Therefore, $30 \mathrm{~s}$ was chosen as the optimal deposition time.
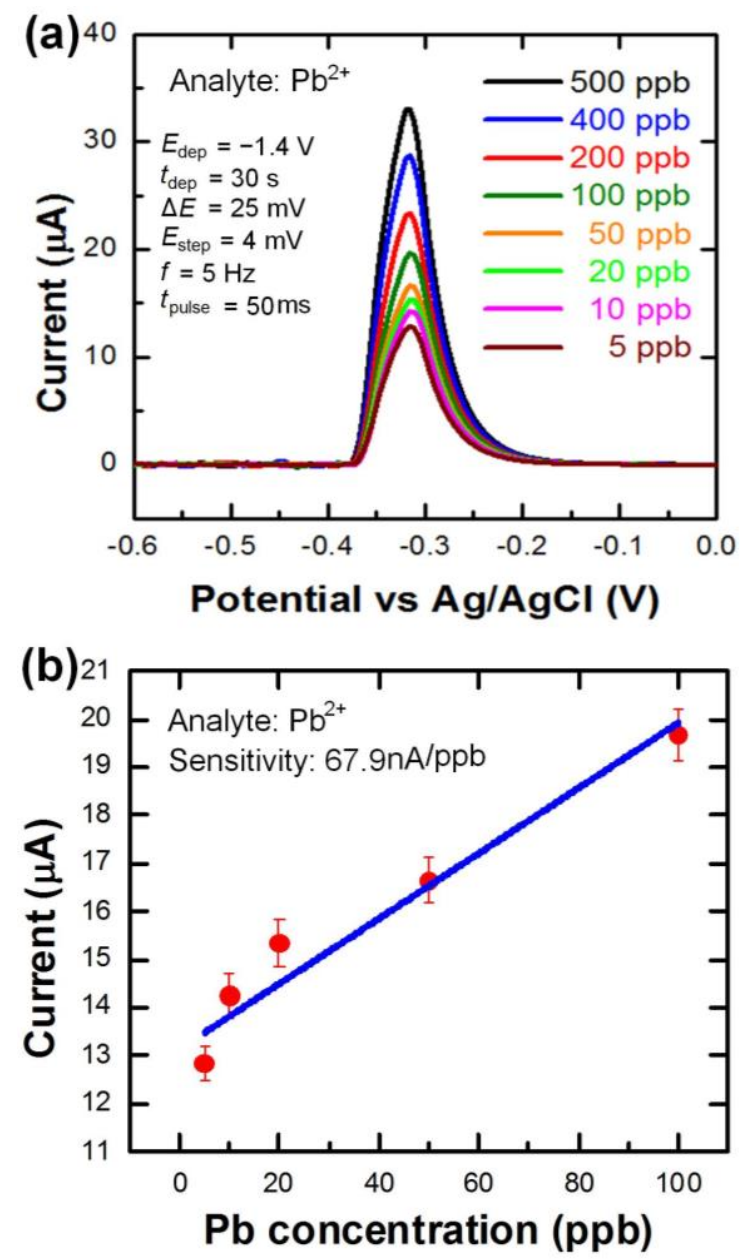

Figure 3:(a) Output current curves from varies concentrations of $\mathrm{Pb}^{2+}$. (b) Calibration curve of (a).

Fig. 3a shows the stripping response of the sensor at different $\mathrm{Pb}^{2+}$ ion concentrations $(5,10,20,50,100,200,400$, and $500 \mathrm{ppb})$. The $\mathrm{Pb}^{2+}$ current peak appeared at $-0.32 \mathrm{~V}$ with respect to the potential of the RE. The corresponding calibration curves exhibited a linear relationship over the concentration range of from $5 \mathrm{ppb}$ to $100 \mathrm{ppb}$. The calculated sensitivity of the sensor is about 67.9 $\mathrm{nA} / \mathrm{ppb}$ (Fig.3b). The LOD of the sensor (defined as the concentration which yield a measure peak with signal-to-noise ratio of 3) was calculated to be about $0.3 \mathrm{ppb}$ of $\mathrm{Pb}^{2+}$. 


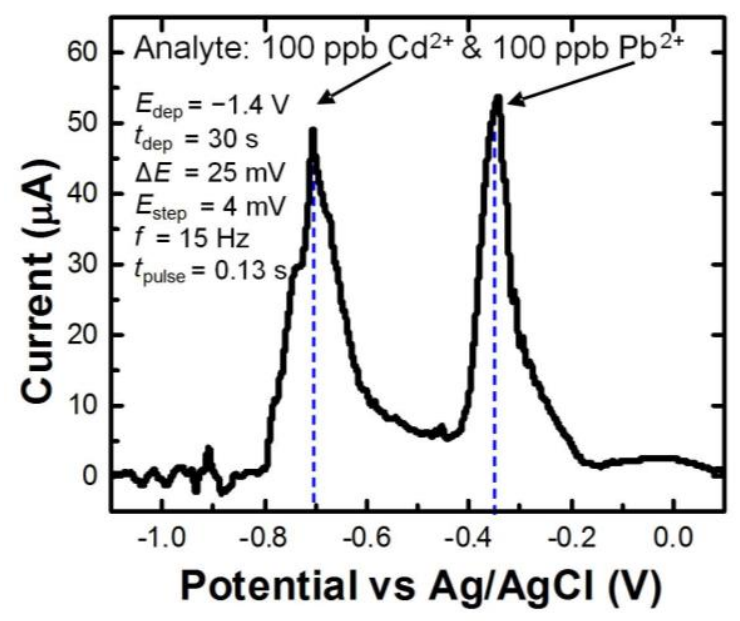

Figure 4: Output current peaks from the mixture of $\mathrm{Cd}^{2+}$ and $\mathrm{Pb}^{2+}$.

Fig. 4 shows the output curve from the mixture of $\mathrm{Cd}^{2+}$ and $\mathrm{Pb}^{2+}$, showing the high selectivity of the sensor. Compared to existing heavy metal ion sensors, the presented sensor offers not only a comparable or better LOD, but also higher compactness and IC compatibility.

\section{CONCLUSION}

We report a miniaturized heavy metal ion sensor able to detect sub-ppb-level $\mathrm{Pb}^{2+}$ in water. The sensor integrates a microfabricated planar $\mathrm{Bi} \mathrm{WE}$, an $\mathrm{Au}$ counter electrode and an $\mathrm{Ag} / \mathrm{AgCl} \mathrm{RE}$ into a microfluidic channel to perform ASV measurement of $\mathrm{Pb}^{2+}$. The developed on-chip sensor exhibited a sharp stripping peak current and a low detect limit for $\mathrm{Pb}^{2+}$. It could be used to monitor many other heavy metal ions in industrial and residential water.

\section{ACKNOWLEDGEMENT}

This work was supported by Analog Devices Inc., Norwood, MA. The authors thank Yingqi Jiang and Frank McNally at Analog Devices Inc., and Junhao Zhu, Azahar Ali, Ranjitha Narayanan, Yang Tian for discussions and technical supports.

\section{REFERENCES}

[1] M.B. Gumpu, S. Sethuraman, U.M. Krishnan, and J.B.B. Rayappan, "A review on detection of heavy metal ions in water-An electrochemical approach", Sensors and Actuators B: Chemical, 213, 515 (2015).

[2] S. Lee, S. Bong, J. Ha, M. Kwak, S.K. Park, and Y. Piao, "Electrochemical deposition of bismuth on activated graphenenafion composite for anodic stripping voltammetric determination of trace heavy metals", Sensors and Actuators B: Chemical, 215, 62 (2015).

[3] J. Wang, J. Lu, S.B. Hocevar, P.A. Farias, and B. Ogoreve, "Bismuth-coated carbon electrodes for anodic stripping voltammetry", Analytical Chemistry, 72, 3218 (2000).

[4] E.P. Achterberg, and C. Braungardt, "Stripping voltammetry for the determination of trace metal speciation and in-situ measurements of trace metal distributions in marine waters", Analytica Chimica Acta, 400, 381 (1999).

[5] G.E. Batley, "Electroanalytical techniques for the determination of heavy metals in seawater", Marine Chemistry, 12, 107 (1983).
[6] A. Giacomino, A. R. Redda, S. Squadrone, M. Rizzi, M. C. Abete, C. La Gioia, T. Rosanna, A. Ornella, and M. Malandrino, "Anodic stripping voltammetry with gold electrodes as an alternative method for the routine determination of mercury in fish. Comparison with spectroscopic approaches", Food Chemistry, 221, 737 (2017).

[7] Z. Nie, C. A. Nijhuis, J. Gong, X. Chen, A. Kumachev, A. W. Martinez, M. Narovlyansky, and G. M. Whitesides, "Electrochemical sensing in paper-based microfluidic devices", Lab on a Chip, 10, 477 (2010).

[8] X. Dai, O. Nekrassova, M.E. Hyde, and R.G. Compton, "Anodic stripping voltammetry of arsenic (III) using gold nanoparticle-modified electrodes", Analytical Chemistry, 76, 5924 (2004).

[9] J. Wang, Ü.A. Kirgöz, J.W. Mo, J. Lu, A.N. Kawde, and A. Muck, "Glassy carbon paste electrodes", Electrochemistry Communications, 3, 203 (2001).

[10] N. Ruecha, N. Rodthongkum, D. M. Cate, J. Volckens, O. Chailapakul, and C. S. Henry, "Sensitive electrochemical sensor using a graphene-polyaniline nanocomposite for simultaneous detection of $\mathrm{Zn}$ (II), Cd (II), and $\mathrm{Pb}$ (II)", Analytica Chimica Acta, 874, 40 (2015).

\section{CONTACT}

* L. Dong, tel: +1-515-294-0388; ldong@iastate.edu 